(c) 2019 IEEE. Personal use of this material is permitted. Permission from IEEE must be obtained for all other uses, in any current or future media, including reprinting/republishing this material for advertising or promotional purposes, creating new collective works, for resale or redistribution to servers or lists, or reuse of any copyrighted component of this work in other works. 


\section{Modelling and Simulation of Handover in Light Fidelity (Li-Fi) Network}

Hieu Danh Huynh \& Kumbesan Sandrasegaran, Faculty of Engineering and IT, University of Technology, Sydney (UTS), New South Wales, Australia

\begin{abstract}
The demand of a faster and more secure wireless communication system leads to the development of a new and innovated network in future. Light Fidelity ( $\mathrm{Li}-\mathrm{Fi}$ ) is being researched to provide a better network for our life facilities. In this communication technology, light from Light Emitting Diodes (LEDs) has been used for data transmission. The purpose of this research work is to investigate the performance of handover algorithms in a Li-Fi network. Two handover algorithms are closest Access Point (AP) and maximum channel gain. MATLAB simulation results are presented to evaluate those two types of handover algorithms and to show the impacts of UE's rotation and movement on handover performance.
\end{abstract}

Index Terms-Light Fidelity, handover algorithms, channel gain.

\section{INTRODUCTION}

Due to the shortage of radio spectrum below $10 \mathrm{GHz}$, the wireless communication system has been considering the radio spectrum above $10 \mathrm{GHz}$. However, communication in higher frequencies also has some problems such as an increase in the path loss, blockages and shadowing. In this scenario, LiFi comes up as one of the best-proposed solutions by using LEDs for high-speed communication [4]. LEDs from Visible Light Communication (VLC) have been used as a medium to deliver communication information in a mobile, networked and high-speed environment like Wi-Fi [5]. Additionally, LiFi system could be built on existing lighting infrastructures. A Li-Fi attocell network also has an ability to minimize interference and provide fully networked wireless connectivity with multiuser access and handover [4]. In Li-Fi network, visible light frequency between 400 and $800 \mathrm{THz}$ (780 and $375 \mathrm{~nm}$ respectively) has been used to carry information for optical transmission and illumination purposes [5].

Some research has been conducted about handover performance in Li-Fi network but without considering UE movement and rotation which happens in an usual UE device. There are four sections in this paper. This section is the first one - Introduction. Section II presents the description of Li-Fi network system followed by the channel gain assessment in section III. Section IV provides simulation results and the conclusion is given in the final section.

\section{OPTICAL SYSTEM DESCRIPTION}

\section{A. Optical system configuration}

Fig. 1 shows the overview of the indoor optical system which contains four LED transmitters (or Access Point - AP) in the four quarters of the room's ceiling and a UE device plays the receiver role on the floor. In this research, the Way Point model [8] has been applied for user movement within a square area with dimension of $\mathbf{b} \times \mathbf{b}\left(m^{2}\right)$.

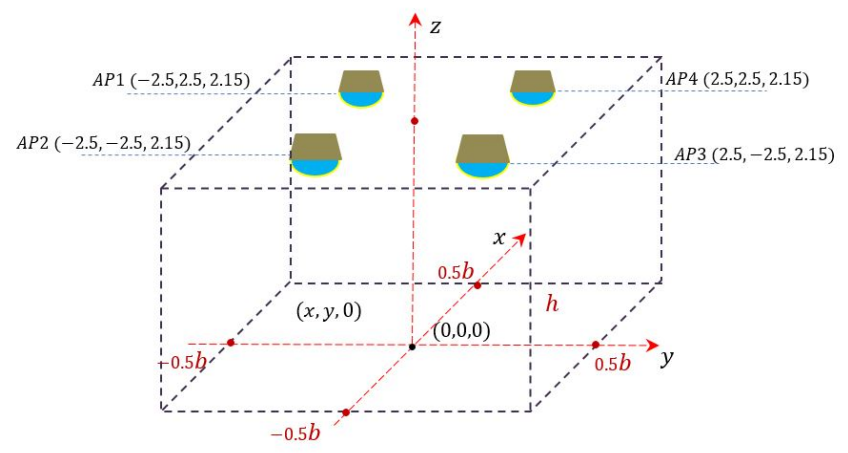

Fig. 1: Downlink geometry in an optical network system.

The assumption in this research are:

1) All LED transmitters emit light vertically downwards.

2) UE device can be rotated in any direction.

3) All LED transmitters have the same power emitted and one unique $\mathrm{AP}$ is chosen for serving the UE depending on its orientation and location.

4) There is no reflection on the wall, ceiling \& floor surfaces.

5) Line-of-sight (LOS) communication channel is considered in this research only.

6) UE device is always on the ground plane of the network area.

The parameters that have been used in this research are given in the following table:

TABLE I: Simulation LED set up

\begin{tabular}{|c|c|}
\hline Name of Parameters & Value \\
\hline \hline Network space (L X W x H) & $10 \mathrm{~m} \mathrm{x} \mathrm{10mx} \mathrm{2.15} \mathrm{m}$ \\
Number of APs & 4 \\
Location of AP1 & $(-2.5,2.5,2.15)$ \\
Location of AP2 & $(-2.5,-2.5,2.15)$ \\
Location of AP3 & $(2.5,-2.5,2.15)$ \\
Location of AP4 & $(2.5,2.5,2.15)$ \\
\hline
\end{tabular}

\section{B. Geometric Orientation Model}

Three angles: $\alpha, \beta$ and $\gamma$ are used to specify the receiver orientation along the $\mathrm{z}, \mathrm{x}$ and $\mathrm{y}$-axis respectively [2]. Fig. 2 describes the UE orientation model about the three axes in "a Cartesian coordinate system" [6]. 


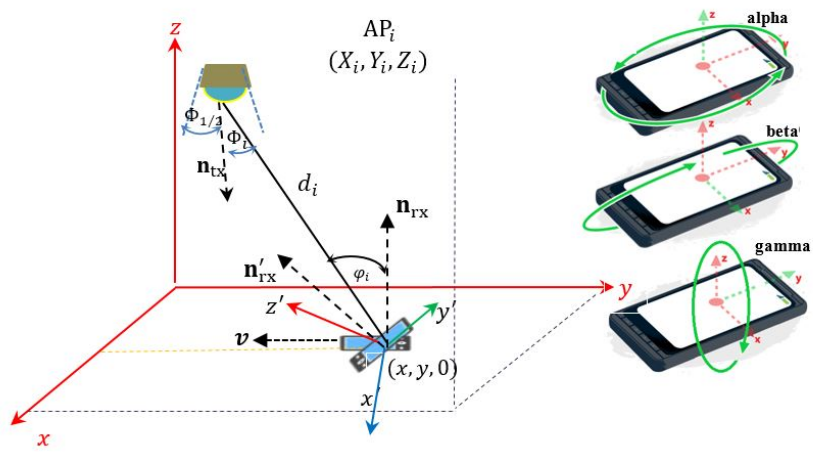

Fig. 2: Receiver orientation modelling based on rotations about three axes [6].

A number of parameters used for specifying the properties of the LED are given in the Table II

TABLE II: Simulation LED Parameters

\begin{tabular}{|c|c|}
\hline Name of Parameters & Value \\
\hline LED half-intensity angle $\phi_{1 / 2}$ & $60^{\circ}$ \\
Receiver FOV $\psi_{c}$ & $90^{\circ}$ \\
Optical filter gain $T_{s}$ & 1 \\
Effective photodetector area $A$ & $1 \times 10^{-4}$ \\
Refractive index m & 1 \\
\hline
\end{tabular}

The angle $\alpha$ describes rotation about the z-axis, and it takes a value between $0^{\circ}$ and $360^{\circ}$ because UE device is assumed that always on the ground plane. The angles $\beta$ and $\gamma$ (both are from $-90^{\circ}$ to $90^{\circ}$ ) is the rotation angle about the $\mathrm{x}$ - and $\mathrm{y}$-axis respectively. The ranges of angles are chosen so as to ensure that the UE is able to communicate with at least one AP. These values are similar to the angles used in [2].

Another parameter is the distance vector between a UE (x, $\mathrm{y}, \mathrm{z})$ and every $A P_{i}\left(X_{i}, Y_{i}, Z_{i}\right)$ and the magnitude of this vector is called the Euclidean distance between $A P_{i}$ and the UE is calculated as follows:

$$
d_{i}=\left\|\boldsymbol{d}_{\boldsymbol{i}}\right\|=\sqrt{\left(X_{i}-x\right)^{2}+\left(Y_{i}-y\right)^{2}+h^{2}}
$$

There are two angles of interest between the UE and AP: $\phi_{i}$ and $\varphi_{i}$ are the angles of radiance with respect to the $\mathrm{z}$ axis on the transmitter plane and the receiver plan for $A P_{i}$ respectively. These angles $\phi_{i}$ and $\varphi_{i}$ are calculated using the rules from geometry:

$$
\begin{gathered}
\cos \phi_{i}=d_{i} \cdot n_{t x} /\left\|d_{i}\right\| \\
\cos \varphi_{i}=-d_{i} \cdot n_{r x} /\left\|d_{i}\right\|
\end{gathered}
$$

where

- $n_{t x}$ and $n_{r x}$ : the normal vectors of the transmitter and receiver planes, respectively

- . and \|\| : the inner product and Euclidean norm operators, respectively.

The optical concentrator gain of the receiver is given by

$$
g\left(\varphi_{i}\right)= \begin{cases}\frac{m^{2}}{\sin \left(\varphi_{c}\right)^{2}} & 0 \leq \varphi_{i} \leq \varphi_{c} \\ 0 & \varphi_{i}>\varphi_{c}\end{cases}
$$

where

- $\mathrm{m}$ : the refractive index

\section{Light Propagation Model}

The irradiance distribution of a LED source is illustrated in the following figure:

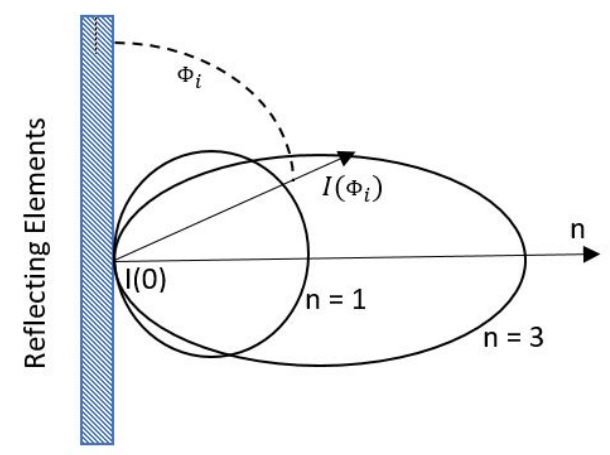

Fig. 3: Lambertian emission pattern for mode $n$

The Lambertian irradiance is defined as [1]:

$$
I\left(\phi_{i}\right)=I(0) \cos ^{n}\left(\phi_{i}\right)
$$

Where, $\mathrm{I}(0)$ is Lambertian irradiance at the centre in $W / \mathrm{m}^{2}$, $\phi_{i}$ is the viewing angle of irradiance, $\mathrm{n}$ is the order of Lambertian irrandiance which can be expressed as [1]:

$$
n=-\frac{1}{\log _{2} \cos \left(\phi_{1 / 2}\right)}
$$

Where $\phi_{1 / 2}$ is the half power angle. Lambertian radiation pattern is expressed as [1]:

$$
R_{0}\left(\phi_{i}\right)=\cos ^{n}\left(\phi_{i}\right) \frac{n+1}{2 \pi}
$$

It is defined as the average power ratio between received and transmitted signal [4].

$$
H_{L O S}= \begin{cases}\frac{A}{d_{i}^{2}} R_{0}\left(\phi_{i}\right) \cos \left(\varphi_{i}\right) & 0 \leq \varphi_{i} \leq \varphi_{c} \\ 0 & \varphi_{i}>\varphi_{c}\end{cases}
$$

And the channel gain at the receiver includes the optical filter gain $T_{s}$ and optical concentrator $\mathrm{g}\left(\varphi_{i}\right)$ integrated in the photodetector

$H_{(}$receiver $)= \begin{cases}\frac{A(n+1)}{d_{i}^{2} 2 \pi} \cos ^{n}\left(\phi_{i}\right) T_{s} \mathrm{~g}\left(\varphi_{i}\right) \cos \left(\varphi_{i}\right) & 0 \leq \varphi_{i} \leq \varphi_{c} \\ 0 & \varphi_{i}>\varphi_{c}\end{cases}$

where

- A: the effective photodetector area

- $T_{s}$ : the receiver's optical filter gain

- $\varphi_{c}$ : the receiver's FOV.

- $\mathrm{g}\left(\varphi_{i}\right)$ : the receiver's optical concentrator gain

- $\phi_{i}$ and $\varphi_{i}$ are the angles of radiance with respect to the $\mathrm{z}$-axis on the transmitter plane and the receiver plan for $A P_{i}$ respectively 


\section{Channel GAIN ASSESSMENT}

In this research, UE moves within the room at a constant speed in a rectangular spiral pattern. UE starts moving from the point $(-5,-5)$ in the easterly direction until reaching the edge of the simulation area. Then this path is repeatedly in the northerly, westerly and southerly directions. After completing one round, UE moves 1 meter inside and this path is repeatedly until reaching the center of the room. It takes 1200 s to complete this spiral path.

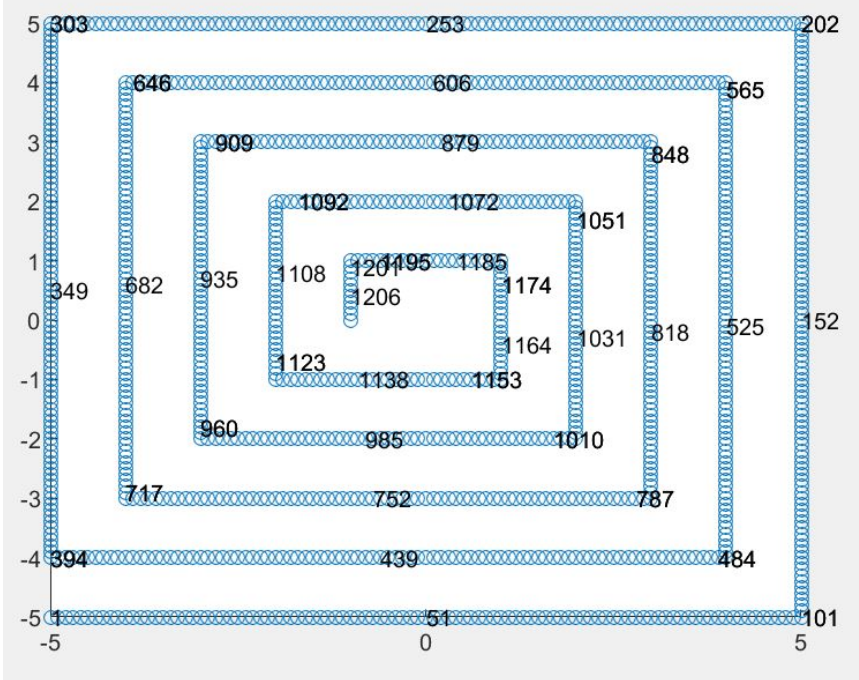

Fig. 4: UE mobility modelling

Two cases are conducted with different value set of $\alpha, \beta$ and $\gamma$. These values are shown in the following table in the following table:

TABLE III: Different value sets of angles

\begin{tabular}{|c|c|c|c|}
\hline Case number & $\alpha$ & $\beta$ & $\gamma$ \\
\hline $\mathbf{1}$ & 0 & 0 & 0 \\
$\mathbf{2}$ & 0 & $45^{\circ}$ & 0 \\
\hline
\end{tabular}

When UE is moving along the rectangular spiral path, with the values of $\alpha, \beta$ and $\gamma$ are fixed for each round, the channel DC gain values are shown in the following figure. Fig. 5 shows the channel gain observed by the UE as it moves in the spiral path facing the default direction $(\alpha=0, \beta=0$ and $\gamma=0)$. Each colour represents channel gain from each AP. Initially when the UE is moving on the perimeter of the rectangle in an anticlockwise direction, the UE is furthest away from the APs and hence the channel gains observed are smallest from all APs. At time $t=0$, the UE is at $(-5,-5)$ and it is closest to AP2 at $(-2.5,-2.5)$ and hence the signal from AP2 (shown in blue) is the strongest signal. Thereafter, between times $\mathrm{t}$ $=51(\mathrm{sec})$ and $\mathrm{t}=100(\mathrm{sec})$, the UE is closest to AP3 at $(2.5,-2.5)$ and hence the signal from AP3 shown in green is the strongest channel gain. At time $t=400$ (sec), the UE has almost completed a full rotation and returned closest to start point and the signal from AP2 shown in blue is the strongest signal. The channel gain at $\mathrm{t}=400(\mathrm{sec})$ is higher than at $\mathrm{t}$ $=0$ because the UE is now closer to AP2 .

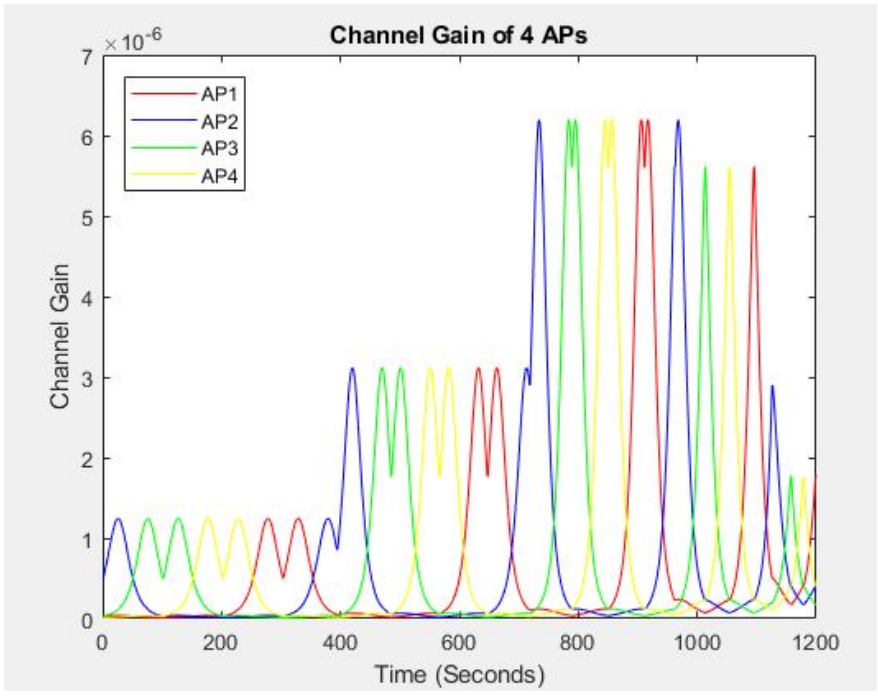

Fig. 5: $\alpha=0, \beta=0$ and $\gamma=0$

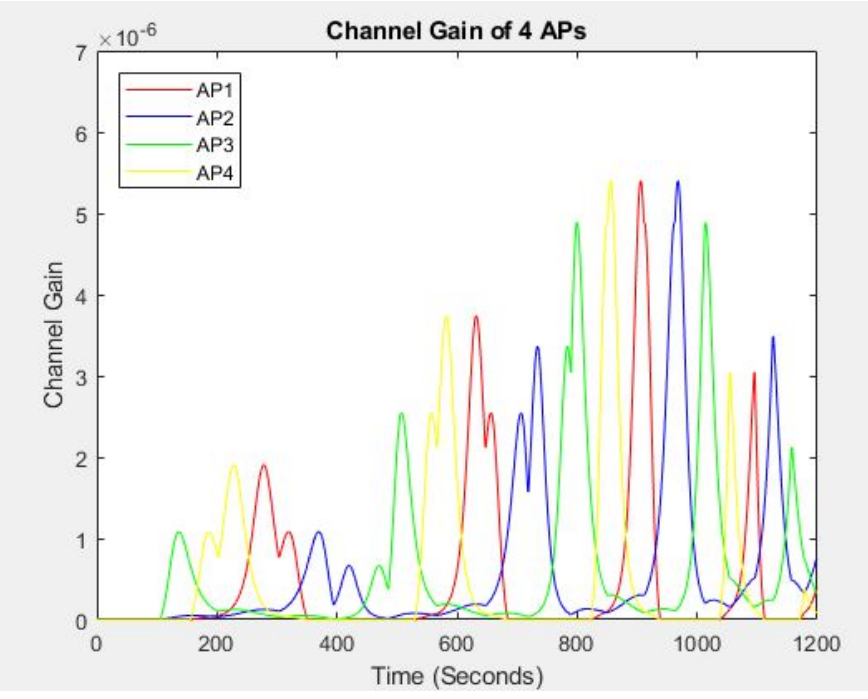

Fig. 6: $\alpha=0, \beta=45^{\circ}$ and $\gamma=0$

After changing values of $\beta$ to $45^{\circ}$ (Fig. 6), the 4 APs' channel gain values reduce slightly. Moreover, the values are equal to zero at the first 100 ( $\mathrm{sec}$ ) where there is no signal received. Similarly, the channel gain values are small in the outer rounds and they becomes larger when getting closer to the room center.

\section{RESUlts}

\section{A. Maximum-channel-gain-based handover decision}

In order to find the serving AP among four APs on the ceiling based on the strongest received signal, the maximum values of channel gain have been selected while UE moves around the network area. These values have been plotted in the following figure: 


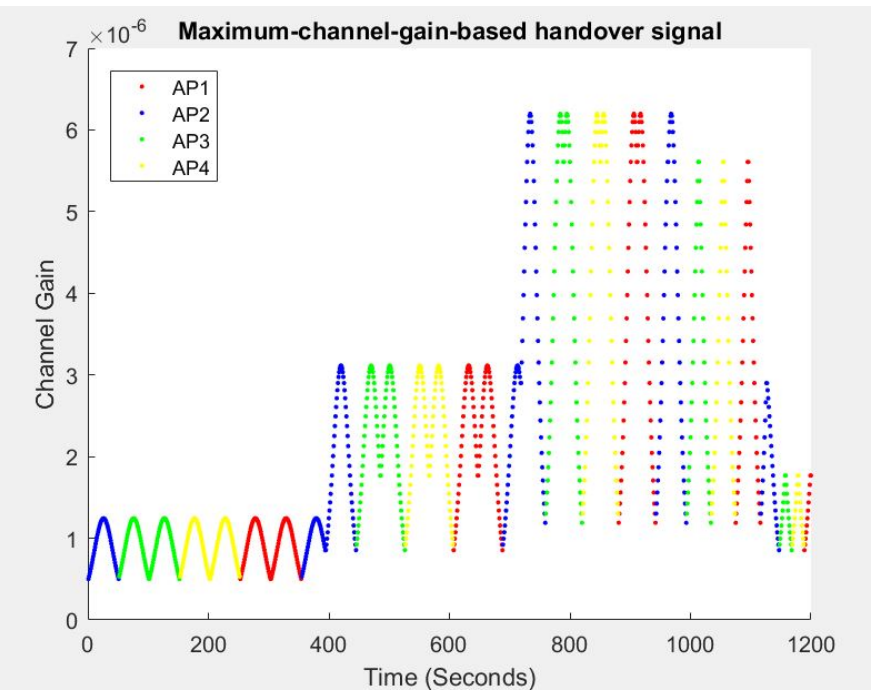

Fig. 7: $\alpha=0, \beta=0$ and $\gamma=0$

When the handover algorithms focuses on choosing the maximum value of channel gain (Fig. 7), the shape of UE's received signal is similar to the maximum values of Fig. 5. Firstly, UE is served by AP2 (blue line) and then by AP3 (green line), AP4 (yellow line) and AP1 (red line) respectively. At the time of 400 ( $\mathrm{sec})$, this value increases more than double previous value as UE has gone to another round inside the room. Then it continues remaining at that level until reaching $750(\mathrm{sec})$.

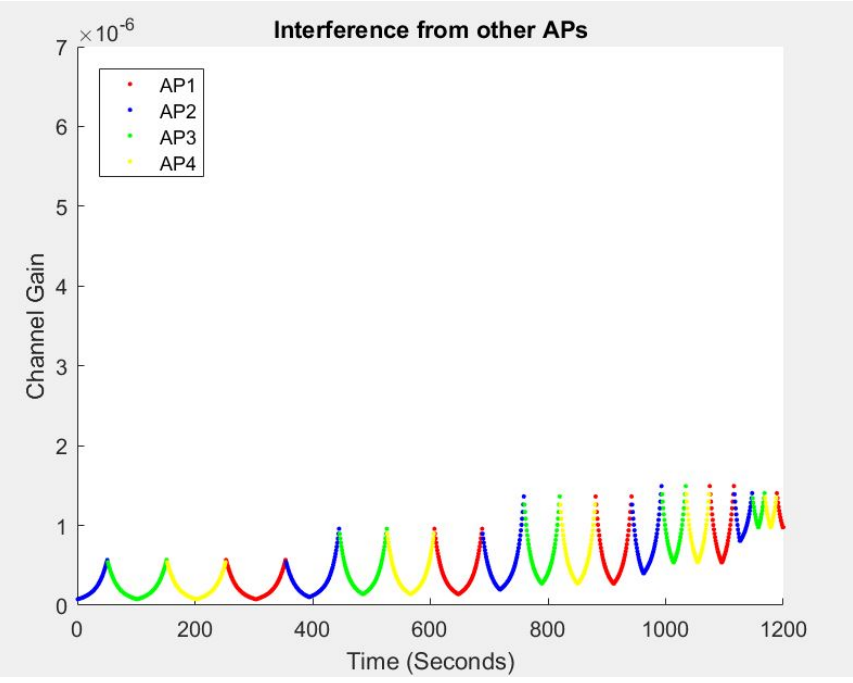

Fig. 8: $\alpha=0, \beta=0$ and $\gamma=0$

If UE is served by one AP which is considered as signal, other 3 APs would be considered as the interference. Fig. 8 shows the total interference of the rest 3 APs when UE is served by any AP. When UE is served by AP2, the blue line shows the total interference of all AP1, AP3 and AP4. And then when it is server by AP3, the channel gain from AP1, AP2 and AP4 will be considered as the interference. In this case, the communication is always possible as the Signal to Interference ratio (SIR) is always greater than 0 . However, the maximum value of SIR is $13.13 \mathrm{~dB}$ with the average value is $8.4 \mathrm{~dB}$ (Table IV).

TABLE IV: Channel gain statistics of maximum-channel-gain-based handover decision when $\alpha=0, \beta=0$ and $\gamma=0$

\begin{tabular}{|c|c|c|c|c|}
\hline $\begin{array}{c}\text { Maximum-channel-gain- } \\
\text { based-handover }\end{array}$ & Maximum & Minimum & Mean & $\begin{array}{c}\text { Standard } \\
\text { deviation }\end{array}$ \\
\hline \hline Gain value $\left(10^{-6}\right)$ & 6.2 & 0.5 & 1.71 & 1.57 \\
Interference value $\left(10^{-6}\right)$ & 1.49 & 0.08 & 0.38 & 0.35 \\
SIR (dB) & 13.13 & -2.16 & 8.4 & 7.11 \\
\hline
\end{tabular}

Table V shows that $94.71 \%$ of the time there is a communication between AP and UE while there is $77.93 \%$ of the time the SIR is larger than $3 \mathrm{~dB}$. During that path, the percentage where SIR is larger than $10 \mathrm{~dB}(23.47 \%)$ is more than one fourth of that for $0 \mathrm{~dB}$.

TABLE V: Overall system performance when $\alpha=0, \beta=0$ and $\gamma=0$

\begin{tabular}{|c|c|c|c|c|}
\hline & SIR $>$ 0dB & SIR $>$ 3dB & SIR $>$ 7dB & SIR $>$ 10dB \\
\hline $\begin{array}{c}\text { Percentage of } \\
\text { time (\%) }\end{array}$ & 94.71 & 77.93 & 56.20 & 23.47 \\
\hline
\end{tabular}

Fig. 9 shows the received signal of UE when the handover decision is based on the maximum channel gains between 4 APs at one time. There is no signal at the period from 0 to $100(\mathrm{sec})$ and then it was chosen among signal from 4 APs to assign signal for UE. And there is $8.8 \%$ of no communication during this path.

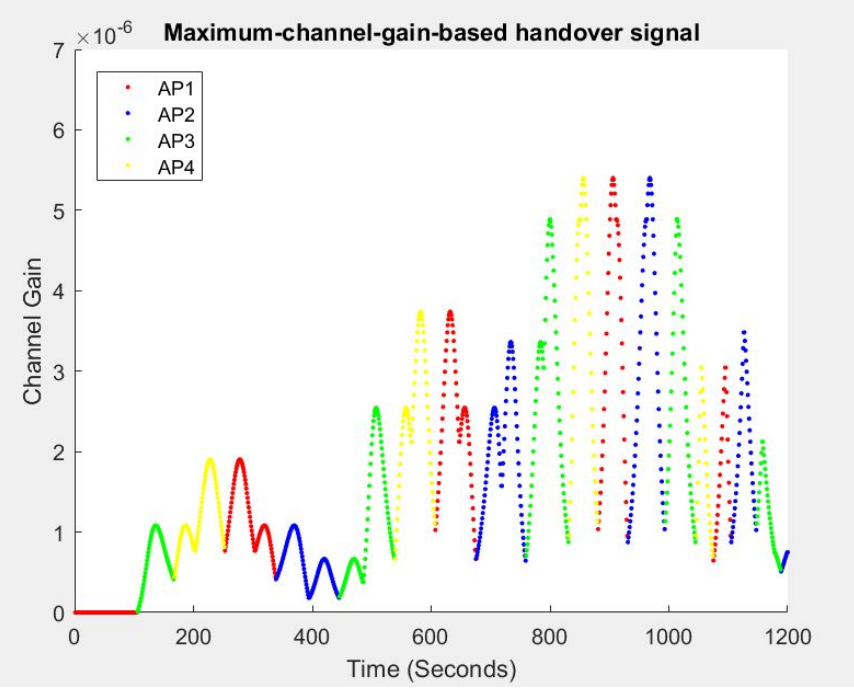

Fig. 9: $\alpha=0, \beta=45^{\circ}$ and $\gamma=0$

From Table VI, although the maximum value of SIR is quite high (17.09) when comparing to previous case but the average value is lower - only 7.82 . 
TABLE VI: Channel gain statistics of maximum-channel-gain-based handover decision when $\alpha=0, \beta=45^{\circ}$ and $\gamma=0$

\begin{tabular}{|c|c|c|c|c|}
\hline $\begin{array}{c}\text { Maximum-channel-gain- } \\
\text { based-handover }\end{array}$ & Maximum & Minimum & Mean & $\begin{array}{c}\text { Standard } \\
\text { deviation }\end{array}$ \\
\hline \hline Gain value $\left(10^{-6}\right)$ & 5.4 & 0 & 1.31 & 1.32 \\
Interference value $\left(10^{-6}\right)$ & 1.47 & 0 & 0.25 & 0.32 \\
SIR $(\mathrm{dB})$ & 17.09 & -3.07 & 7.82 & 5.1 \\
\hline
\end{tabular}

When $\beta=45^{\circ}$ (Table VII), $85.95 \%$ of the time there is a communication between AP and UE while there is $74.38 \%$ of the time the SIR is larger than $3 \mathrm{~dB}$. During this path, the percentage where SIR is larger than $10 \mathrm{~dB}$ is only $32.40 \%$.

TABLE VII: Overall system performance when $\alpha=0, \beta=45^{\circ}$ and $\gamma=0$

\begin{tabular}{|c|c|c|c|c|}
\hline & SIR $>$ 0dB & SIR $>$ 3dB & SIR $>$ 7dB & SIR $>$ 10dB \\
\hline $\begin{array}{c}\text { Percentage of } \\
\text { time (\%) }\end{array}$ & 85.95 & 74.38 & 51.07 & 32.40 \\
\hline
\end{tabular}

\section{B. Closest-AP-based handover decision}

In order to find the serving AP among four APs on the ceiling, the nearest APs have been selected to serve UE while it moves around the network area. These values have been plotted in the following figure:

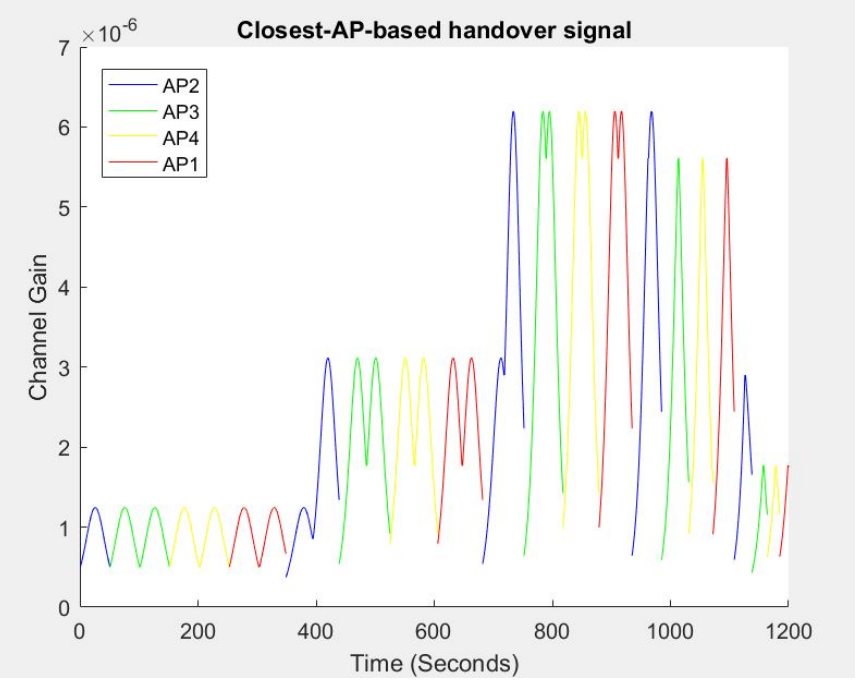

Fig. 10: $\alpha=0, \beta=0$ and $\gamma=0$

When $\alpha=0, \beta=0$ and $\gamma=0$, the UE's signal pattern looks like the patterns of handover algorithms by choosing the maximum channel gain values in Fig. 7. However, there is a discontinuing here as it is chosen regardless of maximum channel gain values. There is a gap in channel gain at 350 (sec) when UE-serving AP is transferred from AP1 to AP2. The maximum and minimum values of SIR are 13.12 and (-17) respectively but the mean value is negative $(-1.1)$.
TABLE VIII: Channel gain statistics of closest-AP-based handover decision

when $\alpha=0, \beta=0$ and $\gamma=0$

\begin{tabular}{|c|c|c|c|c|}
\hline $\begin{array}{c}\text { Closest-AP-based } \\
\text { handover }\end{array}$ & Maximum & Minimum & Mean & $\begin{array}{c}\text { Standard } \\
\text { deviation }\end{array}$ \\
\hline \hline Gain value $\left(10^{-6}\right)$ & 6.2 & 0.01 & 0.14 & 1.21 \\
Interference value $\left(10^{-6}\right)$ & 2.8 & 0.08 & 1.38 & 1.65 \\
SIR & 13.12 & -17 & -1.1 & 6.98 \\
\hline
\end{tabular}

Table IX shows that $47.23 \%$ of the time there is communication between AP and UE while there is only $28.45 \%$ of the time the SIR is larger than $7 \mathrm{~dB}$. During this path, the percentage where SIR is larger than $10 \mathrm{~dB}$ is only $11.58 \%$.

TABLE IX: Overall system performance when $\alpha=0, \beta=0$ and $\gamma=0$

\begin{tabular}{|c|c|c|c|c|}
\hline & SIR $>$ 0dB & SIR $>$ 3dB & SIR $>$ 7dB & SIR $>$ 10dB \\
\hline $\begin{array}{c}\text { Percentage of } \\
\text { time (\%) }\end{array}$ & 47.23 & 39.04 & 28.45 & 11.58 \\
\hline
\end{tabular}

Repeatedly, when the handover algorithm is based on the minimum distance between UE and APs and the $\beta=45^{\circ}$ (Fig. 11), there would be a gap between received signal value while UE is keep moving. This is because the algorithm only choose the channel gain of nearest AP without considering the maximised received signal.

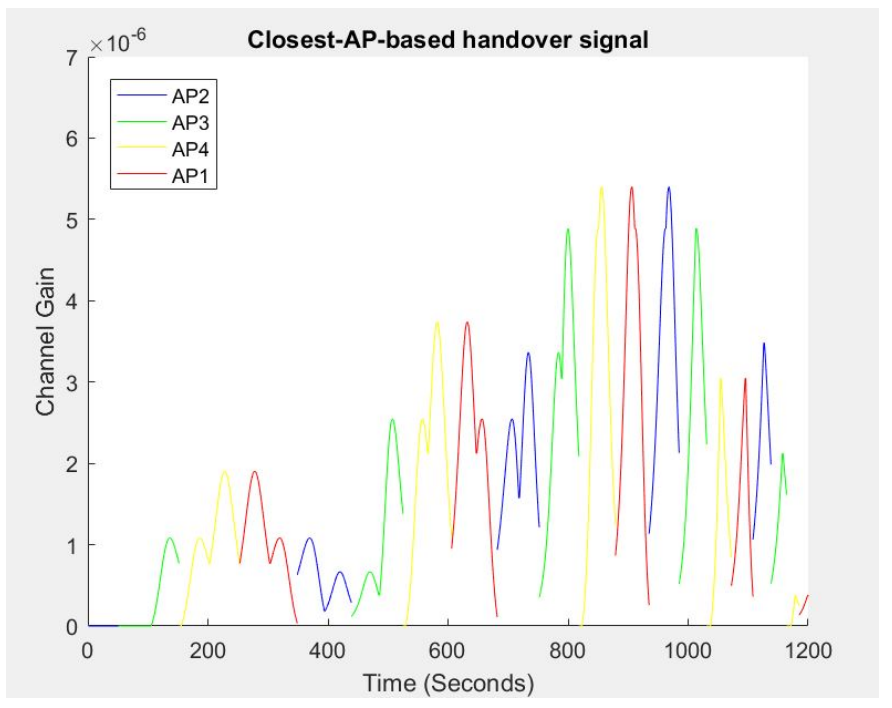

Fig. 11: $\alpha=0, \beta=45^{\circ}$ and $\gamma=0$

There is $52.89 \%$ of no communication during this path which is not considered as the good channel. Additionally, the maximum value of SIR is only 10.13 and the minimum and mean values are 0 . To conclude, when UE is tipping $45^{\circ}$ around $\mathrm{x}$ axis and the handover algorithms based on the nearest APs, the signal does not perform well. 
TABLE X: Channel gain statistics of closest-AP-based handover decision

when $\alpha=0, \beta=45^{\circ}$ and $\gamma=0$

\begin{tabular}{|c|c|c|c|c|}
\hline $\begin{array}{c}\text { Closest-AP-based } \\
\text { handover }\end{array}$ & Maximum & Minimum & Mean & $\begin{array}{c}\text { Standard } \\
\text { deviation }\end{array}$ \\
\hline \hline Gain value $\left(10^{-6}\right)$ & 5.4 & 0 & 0 & 0.974 \\
Interference value $\left(10^{-6}\right)$ & 2.3 & 0 & 1.18 & 1.34 \\
SIR & 10.13 & 0 & 0 & 2.05 \\
\hline
\end{tabular}

Table XI shows that when $\beta=45^{\circ}, 17.95 \%$ of the time there is a communication between AP and UE while there is only $7.03 \%$ of the time the SIR is larger than $7 \mathrm{~dB}$. During this path, the percentage where SIR is larger than $10 \mathrm{~dB}$ is only $0.25 \%$. And this percentage is quite low when comparing to the same handover decision of $\alpha=\beta=\gamma=0$.

TABLE XI: Overall system performance when $\alpha=0, \beta=45^{\circ}$ and $\gamma=0$

\begin{tabular}{|c|c|c|c|c|}
\hline & SIR $>$ 0dB & SIR $>$ 3dB & SIR $>$ 7dB & SIR $>$ 10dB \\
\hline $\begin{array}{c}\text { Percentage of } \\
\text { time (\%) }\end{array}$ & 17.95 & 14.64 & 7.03 & 0.25 \\
\hline
\end{tabular}

\section{Handover Comparison}

TABLE XII: Handover comparison when $\alpha=0, \beta=0$ and $\gamma=0$

\begin{tabular}{|c|c|c|c|c|c|}
\hline & \multicolumn{4}{|c|}{ Closest-AP-based handover } & \multirow[b]{2}{*}{ Total } \\
\hline $\begin{array}{c}\text { Maximum- } \\
\text { channel-gain- } \\
\text { based handover }\end{array}$ & AP1 & AP2 & AP3 & AP4 & \\
\hline AP1 & $24.38 \%$ & $0 \%$ & $0 \%$ & $0 \%$ & $24.38 \%$ \\
\hline AP2 & $0.41 \%$ & $25.21 \%$ & $0 \%$ & $0 \%$ & $25.62 \%$ \\
\hline AP3 & $0 \%$ & $0 \%$ & $25.21 \%$ & $0 \%$ & $25.21 \%$ \\
\hline AP4 & $0 \%$ & $0 \%$ & $0 \%$ & $24.79 \%$ & $24.79 \%$ \\
\hline Total & $24.79 \%$ & $25.21 \%$ & $25.21 \%$ & $24.79 \%$ & $100 \%$ \\
\hline
\end{tabular}

Table XII shows the statistics for serving AP against the closest AP for maximum channel gain handover mechanism. Throughout the simulation interval, the UE is connected to AP1 for $24.38 \%$ of the time and this is also the percentage of UE connected to AP1 when AP1 is the closest AP. Thus in the simulation of maximum channel gain based handover, the UE is connected to the nearest AP for $99.59 \%$ $(24.38+25.21+25.21+24.79)$ of the time and for the remainder $0.41 \%$ of the time, the UE is connected to another AP which is not the closest AP. This could explain the performance improvement for the case of maximum channel gain based handover comparing to the case of nearst AP based handover.

Table XIII shows the statistics for the case of $\beta=45^{\circ}$ where the UE is connected to the nearest AP for only 5.79\% of the time and for the remainder $94.21 \%$ of the time, the UE is connected to another AP which is not the closest AP. From that we could see that UE's rotation affects its channel gain as well as handover decisions.
TABLE XIII: Handover comparison

when $\alpha=0, \beta=45^{\circ}$ and $\gamma=0$

\begin{tabular}{|c|c|c|c|c|c|}
\hline & \multicolumn{4}{|c|}{ Closest-AP-based handover } & \\
\hline \hline $\begin{array}{c}\text { Maximum- } \\
\text { channel-gain- } \\
\text { based handover }\end{array}$ & AP1 & AP2 & AP3 & AP4 & Total \\
\hline \hline AP1 & $0 \%$ & $0 \%$ & $20.74 \%$ & $5.7 \%$ & $\mathbf{2 4 . 3 8 \%}$ \\
AP2 & $24.79 \%$ & $5.79 \%$ & $4.46 \%$ & $0 \%$ & $\mathbf{2 5 . 6 2 \%}$ \\
AP3 & $0 \%$ & $0.33 \%$ & $0 \%$ & $19.09 \%$ & $\mathbf{2 5 . 2 1 \%}$ \\
AP4 & $0 \%$ & $19.09 \%$ & $0 \%$ & $0 \%$ & $\mathbf{2 4 . 7 9 \%}$ \\
\hline \hline Total & $\mathbf{2 4 . 7 9 \%}$ & $\mathbf{2 5 . 2 1 \%}$ & $\mathbf{2 5 . 2 1 \%}$ & $\mathbf{2 4 . 7 9 \%}$ & $\mathbf{1 0 0 \%}$ \\
\hline
\end{tabular}

\section{CONClusion}

The impacts of user rotation and movement have been considered in this research for two types of handover decision: closest-AP-based and maximum-received-signal-based. Overall, we could see that maximum-received-signal-based handover decision performs better than closest-AP-based handover decision: the average channel gain value is 12.7 times larger for the normal case of UE's rotation (from table IV and VIII) and $1.31 \mathrm{~dB}$ higher for the case of $\beta=45^{\circ}$ (from table VI and X). The UE's rotation and movement also have some effects on handover decision causes the received signal to be reduced slighly; however, the percentage of possible communication to be degraded considerably: $8.76 \%$ for the handover decision based on maximum received signal (from table IV and VI) and 2.6 times lower for the handover decision based on closest AP (from table VIII and X). Future works will focus on finding the best handover algorithms in Li-Fi networks.

\section{ACKNOWLEDGEMENTS}

Authors gratefully acknowledge supports by UTS Scholarship and Soltani M. D. Li-Fi R\&D Centre, Institute for Digital Communications, University of Edinburgh, UK.

\section{REFERENCES}

[1] Agarwal A. and Saini G., "SNR Analysis for Visible Light Communication Systems", International Journal of Enngineering Research and Technology (IJERT), vol. 3, issue 10, 2014.

[2] Barthold C., Subbu K. P., and Dantu R., Evaluation GyroscopeEmbedded Mobile Phones, Proc. IEEE Int. Conf. Syst. Man Cybernetics (SMC), Oct. 2011, pp. 16321638.

[3] Ghassemlooy Z., Popoola W. and Rajbhandari S.,Optical Wireless Communications: System and Channel Modelling with MATLAB, Boca Raton, FL: CRC Press, 2013.

[4] Haas H., Yin L. and Wang Y., "What is LiFi?", Journal of Lightwave Technology, vol. 34, no. 6, 2016.

[5] Sathiya T., Divya E. \& Raja S., "Visible Light Communication for Wireless Data Transmission," International Journal of Innovative Research in Electrical, Electronics, Instrumental and Control Engineering, vol. 2, issue 2, 2014.

[6] Soltani M. D., Kazemi H., Safari M. and Haas H., "Handover Modeling for Indoor Li-Fi Cellular Networks: The Effects of Receiver Mobility and Rotation," 2017 IEEE Wireless Communications and Networking Conference (WCNC), San Francisco, CA, 2017, pp. 1-6, 2017.

[7] Bettstetter C., Hartenstein H., and Perez-Costa X., Stochastic Properties of the Random Waypoint Mobility Model, ACM Wireless Netw., vol. 10, no. 5 , pp. 555567 , Sep. 2004 